\title{
UK cancer charity outlines its vision of prevention and treatment
}

Deaths from cancer in the UK - set to soar to 222,000 annually by the year 2020 - could be cut by a third if new treatments and preventive measures are implemented, predicts one of the UK's largest cancer charities, the Imperial Cancer Research Fund (ICRF), in its report Our Vision for Cancer.

The report says that the reorganization of cancer services in Britain, which was announced by the Department of Health in April and is expected to be in place by the turn of the century, could in itself increase survival by as much as 10 percent, by allowing patients to move through the system faster and benefit from treatment by specialists.

The reorganization involves establishing specialist units for treatment of the more common cancers such as breast, bowel and lung cancers in many district hospitals, as well as cancer centres to provide sophisticated diagnostic and treatment facilities and expertise in handling rarer forms of cancer, such as those that affect children and, for example, testicular cancer.

Studies have shown that cure rates for different cancers vary widely in the UK according to where patients are treated. Cure rates for children with leukaemia, lymphoma or bone cancer differ widely from hospital to hospital, and deaths from complications of chemotherapy given to cure testicular cancer in young men are twice as common in some hospitals as in others. The ICRF's report says: "Perhaps around 16,000 lives a year could be saved if the quality of cancer services was uniformly high and the best treatment practices were applied to all."

Most people who develop cancer in Britain are more than 70 years old and the report says that the incidence of cancer will inevitably increase over the next 25 years as the proportion of elderly people in the population rises. According to government projections, the number of people over 65 is expected to rise to 11.8 million by 2020 , up 20 percent from 1992 figures. Deaths from cancer in Britain are forecast to increase from 166,500 this year to 222,000 in 2020 .

Jack Cuzick, a statistician at ICRF, says this figure could be reduced by a third if improvements aimed at preventing or treating some of the more common cancers materialize. For example, by 2020, "efforts that have led to the recent drop in cigarette smoking could cut lung cancer deaths by 30 percent and deaths from bowel cancer could be cut by 40 percent with increasingly sophisticated methods for screening." Screening for bowel cancer could involve a single sigmoidoscopy at about the age of 60 , and presupposes an 80 percent compliance rate and improvements in methods of visualizing the bowel. In addition, Cuzick says, preventive drugs such as tamoxifen, given to women at high risk of breast cancer recurrence, might prevent a quarter of the deaths from this disease.

SHARON KINGMAN London

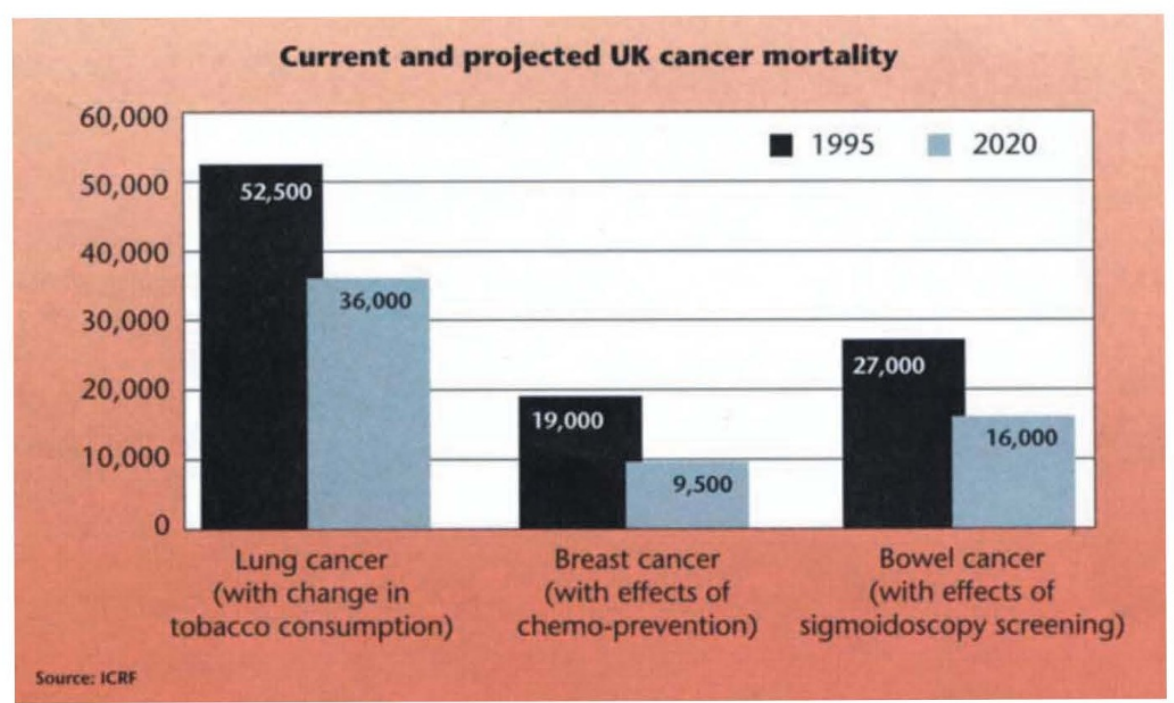

DID YOU KNOW?

Ten cups of Japanese green tea a day keeps the doctor away lapanese green tea, famous for its bitter taste and long tradition, may have therapeutic properties that help keep cancer at bay, according to researchers at Saitama Prefectural Cancer Center, north of Tokyo. The researchers announced their finding at the Annual Japan Cancer Society meeting in Kyoto last month. They pointed out, however, that you have to drink at least ten cups a day for any significant effect. The researchers found that people who drank more than ten cups a day managed to delay the onset of cancer until they were on average 74 years old, whereas people who drank a mere three cups a day succumbed to cancer on average at the age of 65 .

The researchers surveyed 8,552 people over a six-year period and analysed the green tea consumption of 384 who were diagnosed with cancer during that period.

RICHARD NATHAN Tokyo

\section{What's in a soup?}

Mother's chicken soup was, for years, much-maligned as a cure for the common cold until scientists poked their instruments into its chemistry and came up with a positive report. The latest 'kitchen medicine' under the microscope in Australia is toad skin soup - a brew the Chinese have used for thousands of years to treat heart failure.

Thiam Lim, a medical student at the University of Newcastle, New South Wales, has evidence that this delicacy can be a blessing, but also a curse. Lim's poster on the acute effects of Bufo marinus (commonly known as the cane toad) skin extract in vitro was a media favourite at this year's meeting of the Australian and New Zealand

Association for the Advancement of Science.

Using water and ouabain (a cardioglycoside used to treat heart failure) as controls, Lim was able to show that toad skin extract and ouabain had a similar effect on isolated, spontaneously beating guinea pig atria. Both increased heart rate and contractility. At higher concentrations, however, both produced arrhythmia. In a separate experiment, Lim showed that the cane toad extract caused vasoconstriction in human placental vasculature. Lim's conclusion? "The Chinese are probably right in using this in heart failure, but at higher concentrations the patients might get into trouble."

ELIZABETH BAN Sydney 